\title{
A New Theory of Stochastic Inflation
}

\author{
Andrew Matacz * \\ School of Mathematics and Statistics \\ University of Sydney \\ NSW, 2006, Australia
}

\begin{abstract}
The stochastic inflation program is a framework for understanding the dynamics of a quantum scalar field driving an inflationary phase. Though widely used and accepted, there have over recent years been serious criticisms of this theory. In this paper I will present a new theory of stochastic inflation which avoids the problems of the conventional approach. Specifically, the theory can address the quantum-to-classical transition problem, and it will be shown to lead to a dramatic easing of the fine tuning constraints that have plagued inflation theories.
\end{abstract}

98.80.Cq, 05.40.+j, 03.70.+k

*e-mail address: andrewm@maths.su.oz.au 


\section{INTRODUCTION}

The inflationary universe scenario asserts that, at some very early time, the universe went through a de Sitter phase expansion with scale factor $a(t)$ growing as $e^{H t}$. Inflation is needed because it solves the horizon, flatness and monopole problems of the very early universe and also provides a mechanism for the creation of primordial density fluctuations. For these reasons it is an integral part of the standard cosmological model [1].

The inflationary phase is driven by a quantum scalar field with a potential $V(\Phi)$, that can take on many different forms that satisfy the 'slow roll' conditions. Inflationary scenarios fall into two types. In the first, which includes the so-called old and new inflation, the scalar field driving inflation (the inflaton) is assumed to be in thermal equilibrium with the rest of the universe. The universe is assumed to obey the standard hot big bang cosmology in the period preceding and after inflation. In the chaotic inflation scenario these assumptions are relaxed. Instead the inflaton is assumed to be only very weakly coupled to other fields. This makes it possible to choose initial states for the inflaton which are far from equilibrium. The standard big bang cosmology now only applies after the reheating stage of inflation.

In the conventional approach to inflaton dynamics [1], the inflaton field $\Phi$ is first split into a homogeneous piece and an inhomogeneous piece

$$
\Phi(\mathbf{x}, s)=\phi(s)+\psi(\mathbf{x}, s),
$$

where $\phi$ is interpreted as the field $\Phi$ coarse-grained over a volume $\Omega$ :

$$
\phi(s)=\frac{1}{\Omega} \int_{\Omega} \Phi(\mathbf{x}, s) d^{3} \mathbf{x} .
$$

In this description all information on scales smaller than the coarse-graining volume, such as density fluctuations, will be contained in $\psi$. This means the field $\psi$ has a low frequency cutoff in its spectrum. The coarse-graining volume is set by the causal horizon which leads to $\Omega \sim H^{-3}$. We will refer to this $\phi$ as the global order parameter since it is constructed from a coarse-graining volume that is always larger than the observable universe. The dynamics

of the global order parameter is then postulated to obey the classical 'slow roll' equation of motion

$$
\dot{\phi}+\frac{V^{\prime}(\phi)}{3 H}=0
$$

This equation governs the dynamics of $\phi$ which drives the inflationary phase. It is also possible to discuss the generation of primordial density fluctuations using $\psi$. Assuming that $\phi \gg \psi$, it can be shown that $\psi$ is described by a free massless minimally coupled quantum scalar field. During exponential inflation the quantum fluctuations of $\psi$ grow as $\left\langle\psi^{2}\right\rangle \sim H^{3} t$. These quantum fluctuations are then identified with the classical fluctuations which generate primordial density fluctuations [1],2].

Although the conventional theory sketched above is widely accepted, it must be considered problematic for three important reasons:

- it is assumed, without justification, that the global order parameter $\phi$ can be treated as a classical order parameter, and that the quantum fluctuations of $\psi$ are equivalent to classical fluctuations. 
- the fluctuations of $\psi$ lead to an overproduction of primordial density fluctuations. This can only be avoided by unnaturally fine tuning the coupling constants in the inflaton potential.

- the backreaction of $\psi$ on the dynamics of $\phi$ is ignored.

Based on the conventional theory there should be no reason to expect $\phi$ and $\psi$ to behave classically. The action for $\phi$ is non-linear and strongly time dependent. Both of these properties will always generate quantum mechanical coherence over time. Similarly, because $\psi$ is treated as a free quantum field its quantum vacuum state will always remain spatially homogeneous. It is only when these fluctuations become classical that spatial inhomogeneity is generated. Since the conventional theory treats $\phi$ and $\psi$ as independent closed systems it is impossible in principle for it to explain the quantum-to-classical transition of $\phi$ and $\psi$. The purpose of this paper is to develop a more considered approach to inflaton dynamics which can address these three critical issues.

The problems outlined above can all be addressed by a careful application of the principles and techniques of non-equilibrium quantum statistical physics. When we coarse-grain the scalar field as in equation (1.2), what we are effectively doing is splitting our closed quantum system $\Phi$ into a system sector $\phi$ and an environment sector $\psi$. The system sector includes only wavelengths long compared to the coarse-graining scale while the environment sector comprises wavelengths short compared to the coarse-graining scale. By discarding information about the environment sector $\psi$, and assuming that the system and environment interact, we turn the unitary, reversible dynamics of the closed system $\Phi$, into the nonunitary and irreversible dynamics of an open system $\phi$. Thus we have gone from a fundamental theory to an effective theory. The backreaction of any environment sector on a system sector will generally manifest itself by introducing noise, dissipation and renormalization effects into the dynamics of the system [3]. The noise and dissipation will be related by some fluctuation-dissipation relation. Noise will induce diffusion and decoherence (the loss of quantum mechanical coherence). Decoherence is the critical ingredient if we are to dynamically demonstrate the quantum-to-classical transition of an open system.

In this approach our only interest in the environment $\psi$ is its backreaction on the system dynamics. In this case the global order parameter is no longer a suitable system variable because it contains no information about spatial structure within our universe. A system variable that does contain this information can be obtained simply by choosing the apparent rather than the causal horizon as the coarse-graining scale. This corresponds to a coarsegraining volume of $\Omega \sim\left(H e^{H t}\right)^{-3}$. With this averaging volume we will refer to $\phi$ as the local order parameter. The use of a local rather than global order parameter for inflaton dynamics has been strongly advocated by Morikawa [4. In this scheme it is the classical fluctuations $\delta \phi$ of the local order parameter which lead to density fluctuations rather than the quantum fluctuations derived from $\left\langle\psi^{2}\right\rangle$. The new role of the field $\psi$ is to provide a noise source (via backreaction) in the quantum dynamics of the local order parameter. This noise will generate quantum decoherence which is the process that creates the classical fluctuations $\delta \phi$ of the local order parameter. This fundamental conceptual shift regarding the role of $\psi$ is the key to developing a theory that can address the quantum-to-classical transition problem. As a bonus it will be shown that the new theory leads to a dramatic easing of the fine tuning constraints that plague the conventional approach to inflaton dynamics. 
A first attempt to study the backreaction of $\psi$ on the local order parameter $\phi$, can be found in the 'stochastic inflation' program initiated by Starobinsky [5] and further developed by others [6]. This program claims that the semiclassical equation of motion for the local order parameter is given by the Starobinsky equation

$$
\dot{\phi}+\frac{V^{\prime}(\phi)}{3 H}=\frac{H^{3 / 2}}{2 \pi} F_{w}(t),
$$

where $F_{w}(t)$ is a zero mean gaussian white noise source of unit amplitude. The system field equation is thus transformed into a classical Langevin equation with a white noise source. A Fokker-Planck equation can also be derived which depicts the evolution of the probability distribution of the scalar field. Since it was first derived the Starobinsky equation has stimulated numerous studies. Much effort has been devoted to the solution of this stochastic equation for a description of the inflationary transition and the generation of primordial density fluctuations [7]. The same equation forms the theoretical foundation for studies on the very large scale structure of the universe [8]. It has been claimed that the stochastic inflation program can explain the quantum-to-classical transition of the coarsegrained field [9]. A quantum mechanical description, for which the Starobinsky equation is the semiclassical limit, has been developed using methods to quantize classically dissipative systems [10] (this list of references is by no means complete).

In the derivation of the Starobinsky equation, interactions between the local order parameter $\phi$ and its short wavelength environment $\psi$ are derived from the quadratic terms in the scalar field action. Interactions that derive from a self-interacting potential are assumed to be small and are neglected. This procedure cannot be justified because, for free fields, there is no mode-mode coupling and therefore no way for short wavelengths to backreact on long wavelengths. Mode-mode coupling can only be generated by a self-interacting potential. Advocates of stochastic inflation would say that the coupling is generated by the time dependent nature of the system environment split. However it is simply impossible for a physical coupling to be generated by a time dependent choice of what constitutes the system and environment [12, 16, 150]. We would therefore expect there to be, as long as selfinteraction is ignored, no coupling and hence no backreaction of the environment $\psi$ on the local order parameter $\phi$. This implies that classical fluctuations in the local order parameter $\phi$ must vanish for a free theory. We therefore conclude that the Starobinsky equation fails to describe the backreaction of $\psi$ on the local order parameter $\phi$.

A better interpretation for the Starobinsky equation is that it models the conventional approach to inflaton dynamics with a classical stochastic dynamical system. The left hand side is the usual postulated slow roll equation of motion for the global order parameter. The stochastic term is meant to classically model the quantum fluctuations of $\psi$. This interpretation is plausible because solving the free Starobinsky equation gives $\left\langle\phi^{2}\right\rangle \sim H^{3} t$. This is the same as the the growth of quantum fluctuations in $\psi$ in the conventional approach. However, since it is essentially equivalent to the conventional approach, Starobinsky's stochastic inflation program suffers from the same serious problems that were previously outlined.

Several authors have previously made similar criticisms of Starobinsky's stochastic inflation and the conventional inflaton dynamics. Hu and Zhang [12] first questioned the ability of a time-dependent split in generating noise for a free field, and introduced the coarsegrained effective action for a proper treatment of backreaction. Habib [14] demonstrated 
that the Starobinsky theory of stochastic inflation does not address the quantum-to-classical transition despite claims that it does [9]. Hu, Paz and Zhang [16] (see also [17,18]) provided an extensive critique of the Starobinsky theory and developed their own theory of stochastic inflation for the case of a quartic potential. Morikawa [13] pointed out that self-interaction is necessary for the generation of fluctuations, and that a lack of understanding of this point is the cause of the fine tuning problem of inflation. This point has also been emphasized by Calzetta and $\mathrm{Hu}$ [15] in a recent discussion on the problems with conventional inflaton dynamics. See also Morikawa [4] for a general discussion on inflaton dynamics.

Clearly a new theory of stochastic inflation is required which can address the 3 problems of the conventional approach to inflaton dynamics. This new theory will require a fully nonequilibrium quantum mechanical formalism which deals with the statistical nature of mixed states and the dynamics of reduced density matrices. An ideal formalism for this is the Feynman Vernon influence functional [21]. The influence functional describes the averaged effect of the environmental degrees of freedom on the system degrees of freedom to which they are coupled. With the influence functional we can unambiguously identify a noise and dissipation kernel related by some generalized fluctuation-dissipation relation. The formalism leads directly to a propagator for the reduced density matrix which can then be used to derive a master equation. The propagator can be used to study the decoherence process which is the critical part of the quantum-to-classical transition. The master equation can be transformed into a Fokker-Planck type equation for the Wigner function. In the semiclassical limit we have a generalized Langevin equation.

The stochastic inflation program, and indeed many other problems in the early universe [17], fall under the paradigm of quantum Brownian motion (QBM). QBM is one of the two major paradigms of non-equilibrium quantum statistical mechanics which is amenable to detailed analysis (the other being Boltzmann's kinetic theory). The complexity of the problems in cosmology led $\mathrm{Hu}$, Paz and Zhang [20] to first consider a systematic study of QBM in a general environment using the influence functional formalism. The special features associated with a nonohmic bath, or ohmic bath at low temperatures are the appearance of colored noise and nonlocal dissipation. The methodology and viewpoint of QBM have been applied to the analysis of some basic issues in quantum cosmology [23, 18,24, 27, effective field theory [28,29], and the foundation of quantum mechanics, such as the uncertainty principle [30,31] and, most significantly, decoherence [32 36] in the quantum-to-classical transition problem. (See the reviews of [37 39] and references therein for the standard literature on this topic). The QBM models in [20] were further generalized by Hu and Matacz [40] by making the system and bath oscillators the most general time dependent quadratic oscillators, and by considering more general system-bath couplings. The time dependence generates parametric amplification (squeezing) in the oscillators which is precisely how quantum fluctuations of a free field are amplified in the inflationary universe [42]. This formalism allows for a general study of nonequilibrium quantum statistical processes in time dependent backgrounds, as is the case in the early universe.

Using the same methodology as in the previous QBM studies, Hu, Paz and Zhang [16] were the first, based on the system environment split orginally proposed by Starobinsky, to correctly formulate the problem of the backreaction of short wavelength quantum fluctuations $\psi$, on the local order parameter $\phi$. For more recent work of a similar nature see Lombardo and Mazzitelli [19]. These works are based on a rigorous quantum field theory of 
open systems, however they do have some practical shortcomings. The dynamical systems obtained are of a functional nature further complicated by non-local dissipation and colored noise. It is very difficult to analyse the quantum and semiclassical dynamics of these systems. The models are restricted to flat space or conformally coupled fields in a de Sitter phase. For applications to inflation we are mainly interested in minimally coupled scalar fields in a de Sitter phase. The models are very specific, involving a $\lambda \Phi^{4}$ self-interaction. Deriving the renormalized effective action for the long wavelength sector is a complex calculation that can only be done in the context of specific potentials. In inflation there is great interest in a wide variety of potentials. In this case the quantum field theory calculations may be much more difficult to implement than for the $\lambda \Phi^{4}$ case. Because of these problems there has not yet been any detailed investigation of inflaton dynamics based on these quantum field theoretic open systems.

In order to proceed further we need to develop a new simplified theory that overcomes these practical problems. This must be done without compromising a rigorous treatment based firmly on the principles of non-equilibrium quantum statistical physics. Only a theory of this nature can address the failures of conventional inflaton dynamics. In this paper such a theory is developed. The main result is that, for a minimally coupled scalar field in a de Sitter phase, the quantum dynamics of the local order parameter $\phi$ can be described by the relatively simple stochastic quantum mechanical Hamiltonian

$$
H(t)=\frac{p^{2}}{2 e^{3 H t}}+e^{3 H t} V(\phi)-\frac{H^{2}}{8 \pi^{3}} e^{3 H t} V^{\prime \prime}(\phi) F_{c}(t),
$$

where $p=e^{3 H t} \dot{\phi}$ is the canonical momentum and $p$ and $\phi$ obey the usual quantum mechanical commutator. $F_{c}(t)$ is a zero mean gaussian colored noise of unit amplitude with a correlation time of the order $H^{-1}$. This result is valid for a general inflaton potential. The origin of the noise is the backreaction of quantum fluctuations with wavelengths shorter than the coarsegraining scale. The noise is of a multiplicative nature because its origin is the mode-mode coupling induced by the self-interaction of the inflaton. For a free field this theory predicts no noise term. This should be expected from arguments made here and previously [12,16,13,15]. The major simplification is made by ignoring information about spatial correlations between the order parameters of different regions. This allows a description based on a single degree of freedom. Further significant simplification is obtained by invoking the standard slow roll assumptions. This makes it possible to show that the potential renormalization and non-local dissipation terms are negligible.

The approximate semiclassical limit of the quantum open system (1.5) is

$$
\dot{\phi}+\frac{V^{\prime}(\phi)}{3 H}=\frac{H^{1 / 2}}{\sqrt{864} \pi^{3}} V^{\prime \prime \prime}(\phi) F_{w}(t) .
$$

In deriving this equation the slow roll assumptions we used to neglect the inertial term and approximate the colored noise by a white noise $F_{w}$. This equation is no more complicated than the widely used Starobinsky equation (1.4). However it fundamentally differs from it because the noise term vanishes for a free theory. Equation (1.6) is a superior alternative to the Starobinsky equation because it is the semiclassical limit of the quantum open system (1.5). This allows the validity of the semiclassical limit to be dynamically derived by investigating the quantum decoherence generated by the noise in (1.5) (On the other hand, the 
'classicality' of the Starobinsky equation is simply postulated and is not a natural outcome of any quantum open system). The other crucial advantage is that, as we will see, equation (1.6) leads to a dramatic easing of the fine tuning constraints.

\section{STOCHASTIC INFLATION AND QUANTUM BROWNIAN MOTION}

In this section we will see how the dynamics of a coarse-grained scalar field in an expanding universe, can be described in terms of time dependent quantum Brownian motion with non-linear system-bath coupling. We consider a minimally coupled scalar field evolving in a spatially flat background with the metric

$$
d s^{2}=d t^{2}-a^{2}(t) d^{2} \mathbf{x}
$$

The action has the form

$$
S=\int_{t_{i}}^{t} d s \int_{\Omega(s)} d^{3} \mathbf{x} \mathcal{L}(\mathbf{x}, s)
$$

where

$$
\mathcal{L}(\mathbf{x}, s)=a^{3}(s)\left[\frac{1}{2} \dot{\Phi}^{2}(\mathbf{x}, s)-\frac{(\nabla \Phi(\mathbf{x}, s))^{2}}{2 a^{2}(s)}-V(\Phi(\mathbf{x}, s))\right]
$$

We assume $t_{i}$ is our initial time and $a\left(t_{i}\right)=1$.

As is usual in inflation, we split $\Phi$ into a homogeneous piece and an inhomogeneous piece

$$
\Phi(\mathbf{x}, s)=\phi(s)+\psi(\mathbf{x}, s),
$$

and interpret $\phi$ as the field $\Phi$ coarse-grained over a volume $\Omega(s)$. We therefore write

$$
\phi(s)=\frac{1}{\Omega(s)} \int_{\Omega(s)} \Phi(\mathbf{x}, s) d^{3} \mathbf{x}
$$

which will be true as long as

$$
\int_{\Omega(s)} d^{3} \mathbf{x} \psi(\mathbf{x}, s)=\int_{\Omega(s)} d^{3} \mathbf{x} \dot{\psi}(\mathbf{x}, s)=0 .
$$

Assuming $\phi \gg \psi$, the potential term in (2.3) becomes

$$
V(\Phi) \simeq V(\phi)+\psi V^{\prime}(\phi)+\frac{\psi^{2}}{2} V^{\prime \prime}(\phi)
$$

Substituting (2.7) into (2.3) and using (2.6) we find

$$
\begin{aligned}
S & =\int_{t_{i}}^{t} d s \Omega(s) a^{3}(s)\left(\frac{1}{2} \dot{\phi}^{2}(s)-V(\phi)\right)-\frac{1}{2} \int_{t_{i}}^{t} d s a^{3}(s) V^{\prime \prime}(\phi)\left(\int_{\Omega(s)} d^{3} \mathbf{x} \psi^{2}(\mathbf{x}, s)\right) \\
& +\int_{t_{i}}^{t} d s \int_{\Omega(s)} d^{3} \mathbf{x} a^{3}(s)\left[\frac{1}{2} \dot{\psi}^{2}(\mathbf{x}, s)-\frac{(\nabla \psi(\mathbf{x}, s))^{2}}{2 a^{2}(s)}\right] .
\end{aligned}
$$


The coarse-graining breaks the scalar field up into cells of volume $\Omega$, each labeled by a coarse-grained position variable. We have dropped all reference to the position label on $\phi$ since the problem is spatially invariant. By dealing with only one degree of freedom we are ignoring information about spatial correlations between different cells. This is the reason why the middle term in the expansion (2.7) plays no role. Later in this section we justify why we can drop the spatial gradient term of the system sector.

The environment field $\psi$ can be written as

$$
\psi(\mathbf{x})=\sqrt{\frac{2}{\Omega(s)}} \sum_{\mathbf{k}}\left[q_{\mathbf{k}}^{+} \cos \mathbf{k} \cdot \mathbf{x}+q_{\mathbf{k}}^{-} \sin \mathbf{k} \cdot \mathbf{x}\right]
$$

in which case the action (2.8) becomes

$$
\begin{aligned}
S & =\int_{t_{i}}^{t} d s \Omega(s) a^{3}(s)\left[\frac{1}{2} \dot{\phi}^{2}(s)-V(\phi)\right]-\frac{1}{2} \sum_{\sigma}^{+-} \sum_{\mathbf{k}} \int_{t_{i}}^{t} d s a^{3}(s) V^{\prime \prime}(\phi(s)) q_{\mathbf{k}}^{\sigma 2} \\
& +\frac{1}{2} \sum_{\sigma}^{+-} \sum_{\mathbf{k}} \int_{t_{i}}^{t} d s a^{3}(s)\left[\left(\dot{q}_{\mathbf{k}}^{\sigma}\right)^{2}-\frac{k^{2}}{a^{2}(s)} q_{\mathbf{k}}^{\sigma 2}\right]
\end{aligned}
$$

where $k=|\mathbf{k}|$. The action (2.10) is now in the form of a time-dependent one dimensional system, non-linearly coupled to an environment of time dependent harmonic oscillators. This action is a generalization of the well known quantum Brownian motion problem [21,20,40,41. In the next section we will show how the influence functional method can be used to describe the averaged effect of the environment on the quantum dynamics of the system.

\section{A. Low Frequency Cutoff}

We will impose a long wavelength cutoff in the field $\psi$, such that the longest wavelength equals the diameter of the coarse-graining volume. This is necessary since we are interpreting $\phi$ as the field $\Phi$ coarse-grained over the volume $\Omega(s)$. This means that all frequencies less than the cutoff are considered part of the system sector. Since there is no mode-mode coupling for free fields, we cannot expect $\psi$ to couple to $\phi$ in this case. This is clearly true for the action (2.10). Writing the volume element as

$$
\Omega(s)=\frac{4 \pi^{4}}{3 \epsilon^{3} \Lambda^{3}(s)}
$$

we can express the low frequency cutoff as

$$
k_{\min }=\epsilon \Lambda(t)
$$

where $\epsilon$ is an arbitrary dimensionless parameter that scales the coarse-graining volume. An obvious question is how do we choose $k_{\min }$ ?

At a purely technical level we want to choose $\Omega$ large enough so that the spatial gradient term in (2.3) can be neglected for the system sector. The appropriate volume can be deduced from the classical equation of motion for the environment modes. Writing $Q_{k}=q_{k} / a(s)$ we find this equation is 


$$
\partial_{\eta}^{2} Q_{k}+Q_{k}\left(k^{2}-\left(\partial_{\eta}^{2} a\right) / a\right)=0
$$

where $\eta=\int d t / a(t)$ is conformal time. We can see that the environment spectrum is split, with an unstable low frequency sector and a stable effectively flat space sector. Choosing

$$
\Lambda(t)=\sqrt{\left(\partial_{\eta}^{2} a\right) / a}, \quad \epsilon \ll 1
$$

amounts to including all those modes where the spatial gradient is significant in the environment sector.

Detailed results in this paper will be limited to de-Sitter inflation where $a(s)=e^{H s}, \eta=-e^{-H s} / H$ and $t_{i}=0$. In this case (2.14) and (2.12) lead to

$$
k_{\min }=\epsilon H a(t), \quad \epsilon \ll 1 .
$$

This cutoff satisfies our technical requirement. We also have the physical requirement that information about large scale structure is contained in the system sector. This requires $\epsilon$ to be small but finite. Taking $\epsilon \rightarrow 0$ would correspond to an over coarse-grained system that would contain no information of observable scales. The scale of the observable universe today is expected to cross the Hubble radius at least 7 e-foldings after inflation begins [1]. This means that todays horizon scale maps to $k \sim 10^{3} \mathrm{H}$. We can see from (2.15) that after a few e-foldings, scales corresponding to our observable universe are shifted into the system sector. Of course, (2.15) is the same as Starobinsky's choice [5,6] for the low frequency cutoff.

\section{INFLUENCE FUNCTIONAL}

Consider the quantum system described by the action

$$
S[\phi, \mathbf{q}]=S[\phi]+S_{e}[\mathbf{q}]+S_{i n t}[\phi, \mathbf{q}] .
$$

We will take $\phi$ as our system variable and $\mathbf{q}$ to be our environmental variables. Typically the environment has infinite degrees of freedom, denoted here by a bold type. We will briefly review here the Feynman-Vernon influence functional method for deriving the evolution operator [21]. The method provides an easy way to obtain a functional representation for the evolution operator $\mathcal{J}_{r}$ of the reduced density matrix $\hat{\rho}_{r}$.

We are interested in the reduced density matrix defined as

$$
\rho_{r}\left(\phi, \phi^{\prime}\right)=\int_{-\infty}^{+\infty} d \mathbf{q} \int_{-\infty}^{+\infty} d \mathbf{q}^{\prime} \rho\left(\phi, \mathbf{q} ; \phi^{\prime}, \mathbf{q}^{\prime}\right) \delta\left(\mathbf{q}-\mathbf{q}^{\prime}\right),
$$

which is propagated in time by the evolution operator $\mathcal{J}_{r}$

$$
\rho_{r}\left(\phi, \phi^{\prime}, t\right)=\int_{-\infty}^{+\infty} d \phi_{i} \int_{-\infty}^{+\infty} d \phi_{i}^{\prime} \mathcal{J}_{r}\left(\phi, \phi^{\prime}, t \mid \phi_{i}, \phi_{i}^{\prime}, t_{i}\right) \rho_{r}\left(\phi_{i}, \phi_{i}^{\prime}, t_{i}\right) .
$$

By using the functional representation of the full density matrix evolution operator given in (3.2), we can also represent $\mathcal{J}_{r}$ in path integral form. In general, the expression is very 
complicated since the evolution operator $\mathcal{J}_{r}$ depends on the initial state. If we assume that at a given initial time $t_{i}$, the system and the environment are uncorrelated

$$
\hat{\rho}\left(t_{i}\right)=\hat{\rho}_{s}\left(t_{i}\right) \times \hat{\rho}_{e}\left(t_{i}\right),
$$

then the evolution operator for the reduced density matrix does not depend on the initial state of the system and can be written as

$$
\mathcal{J}_{r}\left(\phi_{f}, \phi_{f}^{\prime}, t \mid \phi_{i}, \phi_{i}^{\prime}, t_{i}\right)=\int_{\phi_{i}}^{\phi_{f}} D \phi \int_{\phi_{i}^{\prime}}^{\phi_{f}^{\prime}} D \phi^{\prime} \exp \left\{i\left\{S[\phi]-S\left[\phi^{\prime}\right]\right\}\right\} \mathcal{F}\left[\phi, \phi^{\prime}\right] .
$$

The factor $\mathcal{F}\left[\phi, \phi^{\prime}\right]$, called the 'influence functional', is defined as

$$
\begin{aligned}
\mathcal{F}\left[\phi, \phi^{\prime}\right] & =\exp \left\{i S_{I F}\left[\phi, \phi^{\prime}\right]\right\} \\
& =\operatorname{Tr}\left(\hat{U}\left[\phi_{t, t_{i}}\right] \hat{\rho}_{e}\left(t_{i}\right) \hat{U}^{\dagger}\left[\phi_{t, t_{i}}^{\prime}\right]\right),
\end{aligned}
$$

where $\hat{U}$ is the quantum propagator for the action $S_{e}[\mathbf{q}]+S_{\text {int }}[\phi(s), \mathbf{q}]$ with $\phi(s)$ treated as a time dependent classical forcing term. We have found this form to be very convenient for deriving the influence functional [40,41]. $S_{I F}\left[\phi, \phi^{\prime}\right]$ is the influence action and the effective action for the open quantum system is defined as $S_{\text {eff }}\left[\phi, \phi^{\prime}\right]=S[\phi]-S\left[\phi^{\prime}\right]+S_{I F}\left[\phi, \phi^{\prime}\right]$.

If the interaction term is zero, then it is obvious from its definition, that the influence functional is equal to unity and the influence action is zero. In general, the influence functional is a highly non-local object. Not only does it depend on the time history, but -and this is the more important property- it also irreducibly mixes the two sets of histories in the path integral of (3.5). In those cases where the initial decoupling condition (3.4) is satisfied, the influence functional depends only on the initial state of the environment. The influence functional method can be extended to more general conditions, such as thermal equilibrium between the system and the environment [22], or correlated initial states [21].

The influence action for the open system (2.10), in a de Sitter phase, is derived in appendix A. It has the form

$$
\begin{aligned}
S_{I F}\left[\phi, \phi^{\prime}\right]= & -\int_{0}^{t} d s \Delta(s) f(s)+\int_{0}^{t} d s \int_{0}^{s} d s^{\prime} \Delta(s) \Sigma\left(s^{\prime}\right) \mu\left(s, s^{\prime}\right) \\
& +i \int_{0}^{t} d s \int_{0}^{s} d s^{\prime} \Delta(s) \Delta\left(s^{\prime}\right) \nu\left(s, s^{\prime}\right)
\end{aligned}
$$

where the sum and difference coordinates are

$$
\Sigma(s)=\frac{1}{2}\left(V^{\prime \prime}(\phi(s))+V^{\prime \prime}\left(\phi^{\prime}(s)\right)\right), \quad \Delta(s)=V^{\prime \prime}(\phi(s))-V^{\prime \prime}\left(\phi^{\prime}(s)\right) .
$$

The functions $\nu$ and $\mu$ are known respectively as the noise and dissipation kernels. This is because, as will be shown in section IV, they correspond to colored noise and non-local dissipation. The function $f(s)$ represents a renormalization of the potential. These functions are derived exactly in appendix A.

The approximate form of the noise kernel derived in (A44) is 


$$
\nu\left(s, s^{\prime}\right) \simeq \frac{H^{4} e^{6 H \sigma}}{64 \pi^{6}} \cos (4 \epsilon \sinh (H \delta / 2))+O\left(\epsilon^{2}\right), \quad \epsilon \ll 1 \text { and } \delta<\tau_{c}
$$

where

$$
\delta=s-s^{\prime}, \quad \sigma=\left(s+s^{\prime}\right) / 2 .
$$

As discussed in appendix A, $\tau_{c}$ is the correlation time of the noise kernel which is defined as the time when the argument of the cosine equals $\pi / 4$. The correlation timescale then becomes

$$
\tau_{c}=2 H^{-1} \ln (\pi / 8 \epsilon)
$$

The exact expression for the dissipation kernel is derived in (A46). We do not quote it here since it is a complicated expression that will play little further role. Inspection of (A41) and (A46), the exact noise and dissipation kernels, show that they quickly becomes highly oscillatory when $\delta$ is greater than the correlation time $\tau_{c}$. This means the noise and dissipation kernels are effectively cutoff beyond the correlation time. This oscillatory behaviour is an artifact of the discrete low-frequency cutoff used for the bath. From (A36) the potential renormalization term is

$$
f(s) \simeq \frac{H^{2}}{8 \pi^{2}} e^{3 H s}(1 / 2+\ln (1 / \epsilon)), \quad \epsilon \ll 1 .
$$

One of the most attractive features of this theory is that the approximate noise and dissipation kernels (3.9) and (A48), along with (3.12) have no algebraic dependence on $\epsilon$. This means that physical results will only very weakly depend on $\epsilon$.

\section{A. Local Approximation to the Influence Functional}

We can neglect the potential renormalization term in the influence action (3.7) when

$$
V(\phi) \gg \frac{H^{2}}{8 \pi^{2}} V^{\prime \prime}(\phi) \ln (1 / \epsilon)
$$

This holds when the potential satisfies the standard 'slow roll' condition [1]

$$
\left|V^{\prime \prime}(\phi)\right| \ll 9 H^{2} \simeq 24 \pi V(\phi) / m_{p l}^{2} .
$$

It also holds more generally as long as $H \ll m_{p l}$.

The slow roll conditions of inflation are necessary to ensure that an approximate de-Sitter inflation phase can proceed. In the slow roll limit the dynamical timescale of $\phi$ is much longer than the Hubble timescale, so we expect to be able to make a local approximation to the dynamics. We start by observing that the integrals over $s^{\prime}$ in the influence action (3.7) are strongly suppressed for $\delta>\tau_{c}$. Therefore the lower limit of the integral for $s^{\prime}$ can be cutoff at $s-\tau_{c}$. We can now invoke the slow roll condition. On the time scale $H^{-1}$ we expect $V^{\prime \prime}(\phi)$ to change little, so we can pull out $\Delta\left(s^{\prime}\right)$ and $\Sigma\left(s^{\prime}\right)$ from the integral over $s^{\prime}$ in the influence action. In this case the influence action becomes 


$$
S_{I F}\left[\phi, \phi^{\prime}\right] \simeq \int_{0}^{t} d s \Delta(s) \Sigma(s) \int_{s-\tau_{c}}^{s} d s^{\prime} \mu\left(s, s^{\prime}\right)+i \int_{0}^{t} d s \Delta^{2}(s) \int_{s-\tau_{c}}^{s} d s^{\prime} \nu\left(s, s^{\prime}\right) .
$$

For the noise integral we now find

$$
\begin{aligned}
\int_{s-\tau_{c}}^{s} d s^{\prime} \nu\left(s, s^{\prime}\right) & \simeq \frac{H^{4} e^{3 H s}}{64 \pi^{6}} \int_{s-\tau_{c}}^{s} d s^{\prime} e^{3 H s^{\prime}} \\
& \simeq \frac{H^{3} e^{6 H s}}{192 \pi^{6}}
\end{aligned}
$$

where we used

$$
\cos x \simeq 1
$$

For the dissipation kernel we use the result (A52) derived in appendix A which is

$$
\int_{s-\tau_{c}}^{s} d s^{\prime} \mu\left(s, s^{\prime}\right) \sim \frac{e^{3 H s}}{4 \pi^{2}} \ln (\pi / 8 \epsilon)
$$

The influence action (3.15) now becomes

$$
S_{I F}\left[\phi, \phi^{\prime}\right] \simeq \frac{\ln (\pi / 8 \epsilon)}{8 \pi^{2}} \int_{0}^{t} d s e^{3 H s}\left(V^{\prime \prime 2}(\phi(s))-V^{\prime \prime 2}\left(\phi^{\prime}(s)\right)\right)+\frac{i H^{3}}{192 \pi^{6}} \int_{0}^{t} d s e^{6 H s} \Delta^{2}(s) .
$$

We see that the local approximation to the dissipation kernel has reduced to an effective potential term in the influence action. This term will be much smaller than the system potential when

$$
V(\phi) \gg \ln (\pi / 8 \epsilon) \frac{V^{\prime \prime 2}(\phi)}{8 \pi^{2}}
$$

This will hold whenever the slow roll condition (3.14) is satisfied. As an example we can substitute $V=\lambda \phi^{4}$ into (3.20) which leads to the condition

$$
\lambda \ll \frac{\pi^{2}}{18 \ln (\pi / 8 \epsilon)} .
$$

This is clearly satisfied if $\lambda \ll 1$ which is the standard weak coupling condition. Neglecting the potential term in (3.19), the effective action becomes

$$
S_{e f f}\left[\phi, \phi^{\prime}\right]=S[\phi]-S\left[\phi^{\prime}\right]+\frac{i H^{3}}{192 \pi^{6}} \int_{0}^{t} d s e^{6 H s} \Delta^{2}(s)
$$

with $S[\phi]$ our scaled system action given by (A33) for the case of a de Sitter expansion phase.

In general one must be careful in neglecting non-local dissipation in quantum open systems. Dissipation and noise, connected via a fluctuation-dissipation relation, are twin physical processes that reflect the relation between energy flowing into and out of the system. 
This relation is especially important when one is interested in possible stationary states where a balance is eventually reached. In inflation we have a very flat potential well away from its minimum, and we are only interested in the dynamics over some relatively small finite time. As we showed above it is these very special slow roll conditions of inflation that allowed us to neglect dissipation. This will certainly not be possible during the reheating phase but it should be a good first order approximation to the dynamics in the early slow roll phase.

\section{STOCHASTIC INTERPRETATION OF THE INFLUENCE FUNCTIONAL}

In this section we will show that a stochastic forcing term will generate the imaginary part of the influence action (3.7). Consider the action

$$
S[\phi(s), \xi(s)]=\int_{t_{i}}^{t} d s\left(L(\phi, \dot{\phi}, s)+V^{\prime \prime}(\phi) \xi(s)\right)
$$

where $\xi(s)$ is a zero-mean gaussian stochastic force. This system generates the influence functional

$$
\mathcal{F}\left[\phi, \phi^{\prime}\right]=\left\langle\exp \left[i \int_{t_{i}}^{t} d s \Delta(s) \xi(s)\right]\right\rangle
$$

where the average is understood as a functional integral over $\xi(s)$, weighted by a normalized gaussian probability density functional $\mathcal{P}[\xi(s)]$. The averaging can be performed to give [41]

$$
\mathcal{F}\left[\phi, \phi^{\prime}\right]=\exp \left\{-\int_{t_{i}}^{t} d s \int_{t_{i}}^{s} d s^{\prime} \Delta(s) \Delta\left(s^{\prime}\right) \nu\left(s, s^{\prime}\right)\right\}
$$

where $\nu\left(s, s^{\prime}\right)$ is the second cumulant of the force $\xi$. Clearly then, before averaging over the noise, we can write our effective action derived from (3.7) as

$$
S_{e f f}\left[\phi, \phi^{\prime}, \xi\right]=S[\phi]-S\left[\phi^{\prime}\right]+\int_{t_{i}}^{t} d s \Delta(s)\left[\int_{t_{i}}^{s} d s^{\prime} \Sigma\left(s^{\prime}\right) \mu\left(s, s^{\prime}\right)-f(s)+\xi(s)\right] .
$$

This result can be used to show that that the dynamics of the effective action (3.22), is equivalent to that generated by the quantum mechanical stochastic Hamiltonian

$$
H(t)=\frac{p^{2}}{2 e^{3 H t}}+e^{3 H t} V(\phi)-\frac{H^{3 / 2}}{\sqrt{96} \pi^{3}} e^{3 H t} V^{\prime \prime}(\phi) F_{w}(t),
$$

where $p=e^{3 H t} \dot{\phi}$ is the canonical momentum and $p$ and $\phi$ obey the usual quantum mechanical commutator. $F_{w}(t)$ is a zero mean gaussian white noise with the correlation function

$$
\left\langle F_{w}(t) F_{w}\left(t^{\prime}\right)\right\rangle=\delta\left(t-t^{\prime}\right) .
$$

The white noise Hamiltonian (4.5) is an approximation to 


$$
H(t)=\frac{p^{2}}{2 e^{3 H t}}+e^{3 H t} V(\phi)-\frac{H^{2}}{8 \pi^{3}} e^{3 H t} V^{\prime \prime}(\phi) F_{c}(t)
$$

where $F_{c}(t)$ is a zero mean gaussian colored noise of unit amplitude with the correlation function

$$
\left\langle F_{c}(t) F_{c}\left(t^{\prime}\right)\right\rangle=\cos (4 \epsilon \sinh (H \delta / 2)) .
$$

This follows because the imaginary part of (3.22) is an approximation of the imaginary part of (3.7). Equations (4.5) and (4.7) are the main results of this paper. In the semiclassical limit the system timescale is clearly much greater than the Hubble time due to the slow rolling nature of inflation. In this case a white noise approximation should be reasonable. In the quantum regime it is not so clear that we can approximate (4.7) with (4.5). Numerical simulations will be required to answer this.

\section{A. Semiclassical Limit}

The semiclassical equation of motion for the general effective action (4.4) can be derived using

$$
\left.\frac{\delta\left(S_{e f f}\left[\phi, \phi^{\prime}, \xi\right]\right)}{\delta \Delta_{\phi}(t)}\right|_{\Delta_{\phi}=0}=0
$$

where $\Delta_{\phi}=\phi-\phi^{\prime}$. With the system action (A33) it can be shown that this equation becomes

$$
\left(\ddot{\phi}+3 \frac{\dot{a}}{a} \dot{\phi}+V^{\prime}(\phi)\right) a^{3}(t)+V^{\prime \prime \prime}(s) f(s)-V^{\prime \prime \prime}(\phi) \int_{t_{i}}^{t} \mu(t, s) V^{\prime \prime}(\phi(s)) d s=V^{\prime \prime \prime}(\phi) \xi(t),
$$

where $\left\langle\xi(t) \xi\left(t^{\prime}\right)\right\rangle=\nu\left(t, t^{\prime}\right)$. Under special circumstances $\mu$ tends to the derivative of a delta function which generates local dissipation, hence $\mu$ is referred to as the dissipation kernel. More generally we see that in the semiclassical limit $\mu$ generates non-local dissipation. For the influence action (3.7), the functions $f$ and $\mu$ are unimportant, and the semiclassical equation (4.10) becomes

$$
\ddot{\phi}+3 H \dot{\phi}+V^{\prime}(\phi)=\frac{H^{2}}{8 \pi^{3}} V^{\prime \prime \prime}(\phi) F_{c}(t)
$$

where $F_{c}$ is the zero mean gaussian colored noise with correlator (4.8). This equation is the semiclassical limit of the quantum open system whose dynamics is described by (4.7). Invoking the slow roll approximation (neglecting the $\ddot{\phi}$ ) this equation becomes

$$
\dot{\phi}+\frac{V^{\prime}(\phi)}{3 H}=\frac{H}{24 \pi^{3}} V^{\prime \prime \prime}(\phi) F_{c}(t) .
$$

The neglect of the inertial term in (4.11) is standard practice in inflation when describing the dynamics of the mean field $\phi_{m}$. However it should also be a good approximation for describing the fluctuations $\delta \phi$ about the mean field $\left(\phi_{m}\right.$ is the solution of (1.3)). Because these fluctuations are small we can linearize (4.11) about $\phi_{m}$ to obtain 


$$
\ddot{\delta \phi}+3 H \dot{\delta \phi}+V^{\prime \prime}\left(\phi_{m}\right) \delta \phi=\frac{H^{2}}{8 \pi^{3}} V^{\prime \prime \prime}\left(\phi_{m}\right) F_{c}(t) .
$$

The left hand side of this equation describes a damped simple harmonic oscillator. The standard over-damping condition is the same as the slow roll condition (3.14). Therefore we can neglect the inertial term in (4.13) and conclude that (4.12) should also be a good description for the fluctuations as well as the mean field.

The slow roll conditions allowed us to derive the local approximation (3.22) to the influence functional (3.7). In this approximation (4.12) becomes

$$
\dot{\phi}+\frac{V^{\prime}(\phi)}{3 H}=\frac{H^{1 / 2}}{\sqrt{864} \pi^{3}} V^{\prime \prime \prime}(\phi) F_{w}(t)
$$

where $F_{w}$ is a zero mean gaussian white noise with correlator (4.6). This equation is the semiclassical limit of the result (4.5). It is a superior alternative to the Starobinsky equation (1.4) for reasons that are addressed in the discussion and conclusion section. Since this white noise equation is an approximation to the colored noise equation (4.12), it should be interpreted in the Stratonovich sense.

We have motivated the relatively simple description (4.14) by invoking the slow roll conditions and assuming that a semiclassical description is valid. Ultimately the justification of equation (4.14) will require a detailed study of the quantum dynamics described by the Hamiltonian (4.7). The complicated correlation function (4.8) would make this a difficult task. However it must be emphasized that the complex oscillatory nature of this correlation function is purely an artifact of the discrete low frequency cutoff used for the bath. The only essential 'invariant' information in (4.8) is the correlation time (3.11). Therefore it would be reasonable to investigate the effects of colored noise by replacing the correlator (4.8) with an exponential correlation function with the correlation time (3.11). This correlator generates the simplest type of colored noise dynamics [43] and would make the quantum dynamics described by (4.7) a tractable problem.

\section{AMPLITUDE OF DENSITY FLUCTUATIONS AND THE FINE TUNING PROBLEM}

In this section we will use equation (4.14) to estimate the amplitude of primordial density fluctuations generated by scalar field fluctuations in inflation. We will consider the potential $V=\lambda \phi^{4}$, for which the solution to the slow roll equation (1.3) is

$$
\phi(t)=\phi_{0} \exp \left(-\sqrt{\frac{2 \lambda}{3 \pi}} m_{p l} t\right) .
$$

With this solution it can be shown that the two slow roll conditions of inflation [1] break down for $\phi \sim m_{p l}$. We therefore require $\phi_{0} \gg m_{p l}$. The number of e-folds in the inflationary era is given by [1]

$$
N(\phi)=\frac{8 \pi}{m_{p l}^{2}} \int_{m_{p l}}^{\phi_{0}} d \phi \frac{V(\phi)}{V^{\prime}(\phi)} \simeq \pi \frac{\phi_{0}^{2}}{m_{p l}^{2}} .
$$


A solution to the horizon problem requires $N \geq 60$. This means we require $\phi_{0}$ to be at least several times the Planck mass.

At this point it is useful to compare the dynamical timescale and the Hubble timescale. The dynamical timescale $\tau_{d}$ is found from the solution (5.1) to be

$$
\tau_{d}=\sqrt{\frac{3 \pi}{2 \lambda}} m_{p l}^{-1} .
$$

Using

$$
H^{2}(\phi)=\frac{8 \pi}{3 m_{p l}^{2}} V(\phi)
$$

we find that

$$
\frac{\tau_{d}}{H^{-1}}=2 \pi \phi^{2} / m_{p l}^{2}
$$

When $\phi \gg m_{p l}$ the system timescale is much greater than the Hubble timescale. This provides a explicit example of the condition we assumed to make the local approximation to the influence functional.

So far we have only constrained $\phi_{0}$ to have a lower bound. In chaotic inflation we expect that initially $V \sim m_{p l}^{4}$. This means that that we can only make $\phi_{0} \gg m_{p l}$ if we also correspondingly make $\lambda \ll 1$. In inflation it is the value of the density perturbations which serve to fix a value for $\lambda$, which by the potential constraint also fixes $\phi_{0}$.

The density contrast $\delta \rho / \rho$, that is generated by scalar field fluctuations $\delta \phi$, is approximately given by [1,2]

$$
\frac{\delta \rho}{\rho} \simeq \frac{H}{\dot{\phi}} \delta \phi
$$

Assuming $\phi \gg \delta \phi$, we can linearize equation (4.14) to obtain

$$
\dot{\delta \phi}+\frac{V^{\prime \prime}(\phi)}{3 H} \delta \phi=\frac{H^{1 / 2}}{\sqrt{864} \pi^{3}} V^{\prime \prime \prime}(\phi) F_{w}(t)
$$

We can further simplify this equation by putting $\phi \sim m_{p l}$ which corresponds to the end of inflation. From equation (5.4) the Hubble constant now becomes

$$
H^{2}=\frac{8 \pi}{3} \lambda m_{p l}^{2}
$$

Equation (5.7) is now an Ornstein-Uhlenbeck process for which the stationary distribution gives

$$
\left\langle\delta \phi^{2}\right\rangle=\frac{2 \lambda^{2} m_{p l}^{2}}{9 \pi^{5}}
$$

In this case (5.6) becomes 


$$
\frac{\delta \rho}{\rho} \sim \lambda
$$

Observational constraints then require $\lambda \sim 10^{-6}$. Performing an exactly analogous calculation for the Starobinsky equation (1.4) gives

$$
\frac{\delta \rho}{\rho} \sim \sqrt{\lambda}
$$

which leads to the conventional result $\lambda \sim 10^{-12}$.

Clearly the theory developed here leads to a dramatic easing of the fine-tuning required in this model. The result (5.10) was obtained previously by Calzetta and $\mathrm{Hu}$ [15] using an entirely different approach based on coarse-graining graviton degrees of freedom. They pointed out that this new value of $\lambda$ is consistent with the inflaton taking part in nonabelian gauge interactions with a coupling constant of $10^{-2}$.

\section{DISCUSSION AND CONCLUSION}

In this paper we have argued that the conventional theory of inflaton dynamics [1], along with Starobinsky's theory of stochastic inflation [5, 6], were problematic because they could not explain the quantum-to-classical transition and they predicted an overproduction of primordial density fluctuations. It was concluded that a theory which could address these issues would involve a conceptual shift that comes with being based on the principles of non-equilibrium quantum statistical physics. There have been initial attempts to do this [16,19] using the system environment split originally proposed by Starobinsky [5]. However, as pointed out in the introduction, these models are very complex and this has so far prevented them from being used in a detailed study of inflaton dynamics.

In this paper a new simplified theory of inflaton dynamics was developed. This approach is free of the problems associated with the conventional theory. It is based on the Hamiltonian (4.7) which describes the quantum dynamics of the inflaton which has been coarse-grained over a constant proper volume of a de Sitter inflationary phase. The origin of the noise source is the backreaction of quantum fluctuations with wavelengths shorter than the coarse-graining scale. The noise is of a multiplicative nature because its origin is the mode-mode coupling induced by the self-interaction of the inflaton. For a free field this theory predicts no noise term as we should expect [12,16, 13, 15]. Apart from addressing the problems of the conventional theory, the other essential feature is the relative simplicity of the results (4.7) and (4.14). The major simplification was made by ignoring information about spatial correlations between the order parameters of different regions. This allows a description based on a single degree of freedom. Further significant simplification was obtained by invoking the standard slow roll assumptions. With this we were able to argue that the potential renormalization and non-local dissipation terms were negligible, and that the colored noise could be approximated by a white noise in the semiclassical limit. In this case the theory is independent of the arbitrary parameter $\epsilon$. The correlation time does depend on $\epsilon$ but only logarithmically. The robustness of this theory to the coarse-graining volume, which is parameterized by $\epsilon$, must be considered a major success. Another very important 
feature for applications to inflation is that the theory describes a minimally coupled scalar field with an arbitrary potential.

The slow roll semiclassical equation of motion (4.14), is a superior alternative to the Starobinsky equation for a number of reasons. It is the approximate semiclassical limit of the quantum open system (4.7). This allows the validity of the semiclassical limit to be dynamically derived. The 'classicality' of the Starobinsky equation is simply postulated and is not a natural outcome of any quantum open system. A quantum mechanical description, for which the Starobinsky equation is the semiclassical limit, has been attempted using methods developed some time ago to describe the quantum dynamics of classically dissipative systems [10]. However this amounts to simply 'quantizing' an unjustified semiclassical equation of motion rather than deriving the quantum theory from fundamental physics. In any case, such a procedure is highly suspect because it misconstrues the red-shift term $3 H \dot{\phi}$ in the Starobinsky equation as damping. This repeats an old error in quantum mechanics of confusing a time dependent mass with a real dissipation [11]. The other major advantage is that equation (4.14) leads to a dramatic easing of the fine tuning required in the theory. We showed this for the $\lambda \phi^{4}$ potential. The fine tuning problem is systematic to all potentials of interest in inflation. Similarly, we should expect that equation (4.14) will lead to a large easing of the fine tuning required for these potentials. Finally, the dramatic easing of the fine tuning constraints will make equation (4.14) much simpler to numerically simulate than the Starobinsky equation. Yi and Vishniac (1993) [7] numerically simulated the Starobinsky equation for the $\lambda \phi^{4}$ potential. They used $\lambda=10^{-6}$ in their simulations because the supposedly correct value of $10^{-12}$ was numerically intractable. The former value of $\lambda$ is a natural outcome of the theory presented here.

As for future work, the quantum decoherence generated by the noise term in (4.7) will allow a dynamical investigation of entropy generation and the quantum-to-classical transition. Decoherence studies have been previously attempted [16,19]. However, because these models were very complicated the authors were restricted to numerically calculating the diffusion coefficient in the quantum master equation. Unfortunately this tells us nothing about whether or not enough decoherence will occur in the model to justify a semiclassical description. The critical dynamical aspect to this problem is the competition between the coherence generating non-linear potential and inflation expansion effects, and the decohering stochastic term. This can only be investigated with a full solution of the non-unitary quantum dynamics. If the noise term is strong enough we would hope that decoherence will justify a semiclassical description. However, we must also remember that the noise can not be so large as to destroy the essentially deterministic slow roll dynamics of the local order parameter $\phi$. The inflaton dynamics described by equation (4.7) is the first viable theory to permit a detailed study of the quantum-to-classical of the local order parameter $\phi$. This is the critical process that leads to the generation of classical density fluctuations.

Assuming that the semiclassical description (4.14) is valid, we can use this equation to deduce the amplitude and spectrum of the generated primordial density fluctuations, along with the implications this has for coupling constants of various inflaton potentials. Also of great interest is the generation of any relevant non-gaussian features in the resulting probability distribution of $\phi$ [7], and the implications of equation (4.14) for the very large scale structure of the universe [8]. Till now, these issues have been addressed with the Starobinsky equation as their theoretical foundation. An interesting technical issue is what 
effect relaxing the white noise approximation would have on these results. Investigations into the implications of the theory presented here are in progress.

\section{ACKNOWLEDGMENTS}

I would like to thank the Australia Research Council for their generous support of this research through an Australian Postdoctoral Research Fellowship and an ARC small grant.

\section{APPENDIX A: CALCULATING THE INFLUENCE FUNCTIONAL}

The exact influence functional for the action of the type (2.10) has been previously found to be 41

$$
\mathcal{F}\left[\phi, \phi^{\prime}\right]=\prod_{k}\left\{\alpha_{k}\left[\phi^{\prime}\right] \alpha_{k}^{*}[\phi]-\beta_{k}\left[\phi^{\prime}\right] \beta_{k}^{*}[\phi]\right\}^{-1}
$$

where $\alpha, \beta$ are Bogolubov coefficients which must satisfy the constraint

$$
|\alpha|^{2}-|\beta|^{2}=1
$$

The influence functional (A1) differs from that in 41] because we have here taken into

account the double set of modes in the action (2.10). The Bogolubov coefficients are solutions of the equations

$$
\begin{aligned}
\dot{\alpha} & =-i g \beta-i h \alpha \\
\dot{\beta} & =i h \beta+i g \alpha
\end{aligned}
$$

where

$$
\begin{aligned}
& g(s)=\frac{1}{2}\left(\frac{a^{3}(s) \omega^{2}(s)}{k}-\frac{k}{a^{3}(s)}\right) \\
& h(s)=\frac{1}{2}\left(\frac{k}{a^{3}(s)}+\frac{a^{3}(s) \omega^{2}(s)}{k}\right),
\end{aligned}
$$

and

$$
\omega^{2}(s)=k^{2} / a^{2}+V^{\prime \prime}(\phi(s)) .
$$

We must have $\alpha=1$ and $\beta=0$ at $t_{i}$ as our initial conditions.

Defining

$$
\mathbf{U}\left(t, t_{i}\right)=\left(\begin{array}{cc}
\alpha\left[\phi_{t, t_{i}}\right] & \beta^{*}\left[\phi_{t, t_{i}}\right] \\
\beta\left[\phi_{t, t_{i}}\right] & \alpha^{*}\left[\phi_{t, t_{i}}\right]
\end{array}\right),
$$

the solution of (A3-4) can be written as 


$$
\mathbf{U}\left(t, t_{i}\right)=\mathcal{T} \exp \left(-i \int_{t_{i}}^{t} d s \mathbf{u}(s)\right)
$$

where

$$
\mathbf{u}(s)=\left(\begin{array}{cc}
h(s) & g(s) \\
-g(s) & -h(s)
\end{array}\right)
$$

We will write

$$
\mathbf{u}(\mathbf{s})=\mathbf{u}_{\mathbf{0}}(\mathbf{s})+\mathbf{u}_{\mathbf{1}}(\mathbf{s})
$$

where

$$
\mathbf{u}_{\mathbf{1}}(s)=\frac{a^{3}(s) V^{\prime \prime}(\phi(s))}{2 k}\left(\begin{array}{cc}
1 & 1 \\
-1 & -1
\end{array}\right)
$$

In this case we have

$$
\mathbf{U}\left(t, t_{i}\right)=\mathbf{U}_{0}\left(t, t_{i}\right) \mathbf{U}_{1}\left(t, t_{i}\right)
$$

where $\mathbf{U}_{0}\left(t, t_{i}\right)$ is $\mathbf{U}\left(t, t_{i}\right)$ evaluated at $V^{\prime \prime}(\phi)=0$. The equation of motion for $\mathbf{U}_{1}$ is

$$
\dot{\mathbf{U}}_{1}\left(s, t_{i}\right)=-i\left(\mathbf{U}_{0}^{-1}\left(s, t_{i}\right) \mathbf{u}_{1}(s) \mathbf{U}_{0}\left(s, t_{i}\right)\right) \mathbf{U}_{1}\left(s, t_{i}\right)
$$

where $\mathbf{U}^{-1}$ denotes the inverse matrix. This inverse is

$$
\mathbf{U}^{-1}\left(t, t_{i}\right)=\left(\begin{array}{cc}
\alpha^{*}\left[\phi_{t, t_{i}}\right] & -\beta^{*}\left[\phi_{t, t_{i}}\right] \\
-\beta\left[\phi_{t, t_{i}}\right] & \alpha\left[\phi_{t, t_{i}}\right]
\end{array}\right)
$$

because of the constraint (A2). From (A13) and (A8) we have

$$
\begin{aligned}
& \alpha\left[\phi_{t, t_{i}}\right]=\alpha_{0}\left(t, t_{i}\right) \alpha_{1}\left[\phi_{t, t_{i}}\right]+\beta_{0}^{*}\left(t, t_{i}\right) \beta_{1}\left[\phi_{t, t_{i}}\right] \\
& \beta\left[\phi_{t, t_{i}}\right]=\beta_{0}\left(t, t_{i}\right) \alpha_{1}\left[\phi_{t, t_{i}}\right]+\alpha_{0}^{*}\left(t, t_{i}\right) \beta_{1}\left[\phi_{t, t_{i}}\right] .
\end{aligned}
$$

Substituting this into the influence functional (A1), and using the constraint (A2), we find

$$
\mathcal{F}\left[\phi, \phi^{\prime}\right]=\prod_{k}\left\{\alpha_{1}\left[\phi^{\prime}\right] \alpha_{1}^{*}[\phi]-\beta_{1}\left[\phi^{\prime}\right] \beta_{1}^{*}[\phi]\right\}^{-1}
$$

where we have dropped the $k$ subscript on the Bogolubov coefficients.

To second order in $V^{\prime \prime}$ the solution to (A14) is

$$
\begin{aligned}
\mathbf{U}_{1}\left(t, t_{i}\right) & =1-i \int_{t_{i}}^{t} d s \mathbf{U}_{0}^{-1}\left(s, t_{i}\right) \mathbf{u}_{1}(s) \mathbf{U}_{0}\left(s, t_{i}\right) \\
& -\int_{t_{i}}^{t} d s \int_{t_{i}}^{s} d s^{\prime} \mathbf{U}_{0}^{-1}\left(s, t_{i}\right) \mathbf{u}_{1}(s) \mathbf{U}_{0}\left(s, t_{i}\right) \mathbf{U}_{0}^{-1}\left(s^{\prime}, t_{i}\right) \mathbf{u}_{1}\left(s^{\prime}\right) \mathbf{U}_{0}\left(s^{\prime}, t_{i}\right) .
\end{aligned}
$$

Using this solution and (A18), the influence action becomes 


$$
\begin{aligned}
S_{I F}\left[\phi, \phi^{\prime}\right] & =\sum_{k}\left\{-\frac{1}{2 k} \int_{t_{i}}^{t} d s a^{3}(s) \Delta(s) X_{k}(s) X_{k}^{*}(s)\right. \\
& +\frac{i}{8 k^{2}} \int_{t_{i}}^{t} d s \int_{t_{i}}^{s} d s^{\prime} a^{3}(s) a^{3}\left(s^{\prime}\right) \Delta(s) \Delta\left(s^{\prime}\right)\left[X_{k}^{* 2}(s) X_{k}^{2}\left(s^{\prime}\right)+X_{k}^{2}(s) X_{k}^{* 2}\left(s^{\prime}\right)\right] \\
& \left.-\frac{i}{4 k^{2}} \int_{t_{i}}^{t} d s \int_{t_{i}}^{s} d s^{\prime} a^{3}(s) a^{3}\left(s^{\prime}\right) \Delta(s) \Sigma\left(s^{\prime}\right)\left[X_{k}^{* 2}(s) X_{k}^{2}\left(s^{\prime}\right)-X_{k}^{2}(s) X_{k}^{* 2}\left(s^{\prime}\right)\right]\right\}
\end{aligned}
$$

where

$$
\Delta(s)=V^{\prime \prime}(\phi(s))-V^{\prime \prime}\left(\phi^{\prime}(s)\right), \quad \Sigma(s)=\frac{1}{2}\left[V^{\prime \prime}(\phi(s))+V^{\prime \prime}\left(\phi^{\prime}(s)\right)\right]
$$

and

$$
X_{k}(s)=\alpha_{0}\left(s, t_{i}\right)+\beta_{0}\left(s, t_{i}\right)
$$

Using (A3-4) we can show that (A22) obeys the classical equation of motion

$$
\ddot{X}+3 \frac{\dot{a}}{a} \dot{X}+\frac{k^{2}}{a^{2}} X=0
$$

subject to the initial conditions

$$
X\left(t_{i}\right)=1, \quad \dot{X}\left(t_{i}\right)=-i k .
$$

These initial conditions ensure $\alpha\left(t_{i}\right)=1$ and $\beta\left(t_{i}\right)=0$.

Taking the continuum limit we have

$$
\sum_{k} \rightarrow \frac{\Omega}{4 \pi^{2}} \int_{k_{\min }}^{\infty} k^{2} d k
$$

where we adopt the notation that $\Omega$ is a symmetric function of $s$ and $s^{\prime}$, i.e. $\Omega^{2} \equiv \Omega(s) \Omega\left(s^{\prime}\right)$. In this case the influence action (A20) becomes

$$
\begin{aligned}
S_{I F}\left[\phi, \phi^{\prime}\right]= & -\int_{t_{i}}^{t} d s \Delta(s) f(s)+\int_{t_{i}}^{t} d s \int_{t_{i}}^{s} d s^{\prime} \Delta(s) \Sigma\left(s^{\prime}\right) \mu\left(s, s^{\prime}\right) \\
& +i \int_{t_{i}}^{t} d s \int_{t_{i}}^{s} d s^{\prime} \Delta(s) \Delta\left(s^{\prime}\right) \nu\left(s, s^{\prime}\right)
\end{aligned}
$$

where

$$
\begin{gathered}
f(s)=a^{3}(s) \frac{\Omega}{8 \pi^{2}} \int_{k_{m i n}}^{\infty} d k k X_{k}(s) X_{k}^{*}(s) \\
\nu\left(s, s^{\prime}\right)=a^{3}(s) a^{3}\left(s^{\prime}\right) \frac{\Omega}{32 \pi^{2}} \int_{k_{\text {min }}}^{\infty} d k\left[X_{k}^{* 2}(s) X_{k}^{2}\left(s^{\prime}\right)+X_{k}^{2}(s) X_{k}^{* 2}\left(s^{\prime}\right)\right]
\end{gathered}
$$

and

$$
\mu\left(s, s^{\prime}\right)=-i a^{3}(s) a^{3}\left(s^{\prime}\right) \frac{\Omega}{16 \pi^{2}} \int_{k_{\min }}^{\infty} d k\left[X_{k}^{* 2}(s) X_{k}^{2}\left(s^{\prime}\right)-X_{k}^{2}(s) X_{k}^{* 2}\left(s^{\prime}\right)\right]
$$


From the action (2.8) we see that it is $\psi^{2}$ that couples to the system. The truncation of the solution (A19) amounts to assuming that $\psi^{2}$ generates a gaussian noise source on the system. This is proved in section 4 . The gaussian property ensures that only the first two cumulants of the stochastic process generated by $\psi^{2}$ appears in the influence functional. The influence functional can be expressed in the alternative form [41]

$$
\begin{aligned}
f(s) & \sim\left\langle\psi^{2}(s)\right\rangle \\
\mu\left(s, s^{\prime}\right) & \sim\left\langle\psi^{2}(s) \psi^{2}\left(s^{\prime}\right)\right\rangle-\left\langle\psi^{2}\left(s^{\prime}\right) \psi^{2}(s)\right\rangle \\
\nu\left(s, s^{\prime}\right) & \sim\left\langle\psi^{2}(s) \psi^{2}\left(s^{\prime}\right)\right\rangle+\left\langle\psi^{2}\left(s^{\prime}\right) \psi^{2}(s)\right\rangle-2\left\langle\psi^{2}(s)\right\rangle\left\langle\psi^{2}\left(s^{\prime}\right)\right\rangle
\end{aligned}
$$

which makes this clearer. For a gaussian process all higher order cumulants vanish because all higher order moments can be expressed in terms of first and second moments. Clearly then, the truncation of the solution (A19) is an equivalent approximation to writing all higher order moments of $\psi^{2}$ in terms of first and second moments.

Our quantum field theory has the commutator

$$
\left[\Phi(\mathbf{x}), P_{\Phi}(\mathbf{y})\right]=i \hbar \delta(\mathbf{x}-\mathbf{y})
$$

where $P_{\Phi}(\mathbf{y})$ is the canonical field momentum derived from the action (2.3). Using this commutator we can show that our coarse-grained field (2.5), and a similarly defined coarsegrained canonical momentum, will obey the usual single particle quantum mechanical commutator only after we perform the scaling

$$
\hbar \rightarrow \Omega(s) \hbar
$$

on the Planck constant. This is a natural consequence of reducing a quantum field theoretic problem to a quantum mechanical problem and has been discussed previously [10]. We have set $\hbar=1$ in this paper, but the scaling (A32) is equivalent to scaling the effective action (4.4) by $\Omega(s)$. This gives the new system action

$$
S[\phi]=\int_{t_{i}}^{t} d s a^{3}(s)\left[\frac{1}{2} \dot{\phi}^{2}(s)-V(\phi)\right]
$$

and it requires that we must scale the functions (A27-A29) as

$$
f(s) \rightarrow \Omega^{-1} f(s), \quad \mu\left(s, s^{\prime}\right) \rightarrow \Omega^{-1} \mu\left(s, s^{\prime}\right), \quad \nu\left(s, s^{\prime}\right) \rightarrow \Omega^{-2} \nu\left(s, s^{\prime}\right) .
$$

Equation (A23) is identical to (2.13) when we scale $X$ by the scale factor and transform to conformal time. For a de Sitter phase, the solution of (2.13) leads to

$$
X_{k}(s)=\frac{e^{-i k \eta}}{a(s)}\left(1-\frac{i}{k \eta}\right) .
$$

Strictly speaking an appropriate linear combination of these complex mode functions are required to satisfy the initial conditions (A24). However the time dependent low frequency cutoff ensures that any dependence on the initial condition is a transient effect. So the description based on the mode function (A35) is accurate on the Hubble timescale. With 
(A35) we are now in a position to calculate (A27-A29) using (2.15) as the low frequency cutoff.

We will first consider the function $f(s)$. This function is ultraviolet divergent which is not surprising since $f \sim\left\langle\psi^{2}\right\rangle$. We will adopt the usual non-rigorous renormalization procedure here simply by imposing the inflationary ultraviolet cutoff $k=H a$ (see Brandenberger (1984) [2] and Habib [14]). This cutoff is determined by demanding that the energy density of inflaton fluctuations be less than the potential (which is supposed to dominate), and that the inflaton fluctuations can be taken to be in the vacuum state. This is discussed by Liddle and Lyth [1]. We must also perform the scaling (A34) on (A27) which eliminates the volume factor $\Omega$ from (A27). We therefore find

$$
\begin{aligned}
f(s) & =\frac{a(s)}{8 \pi^{2}} \int_{\epsilon H a}^{H a} d k\left(k+\frac{1}{k \eta^{2}}\right) \\
& =\frac{H^{2}}{8 \pi^{2}} e^{3 H s}(1 / 2+\ln (1 / \epsilon)), \quad \epsilon \ll 1 .
\end{aligned}
$$

The small $\epsilon$ limit is necessary in order to ignore the spatial gradient term in the system sector. This was shown in section 2 where it was also discussed that we require $\epsilon$ to be small but finite. We are therefore never faced with any problems associated with infrared divergences.

After performing the scaling (A34) on (A28) and substituting (A35) into (A28) we find that the noise kernel becomes

$$
\begin{aligned}
\nu\left(s, s^{\prime}\right) & =\frac{a(s) a\left(s^{\prime}\right)}{16 \Omega \pi^{2}} \int_{\epsilon H a}^{\infty} d k\left[\cos 2 k \delta_{\eta}\left(1+\frac{2}{k^{2} \eta \eta^{\prime}}-\frac{\delta_{\eta}^{2}}{k^{2} \eta^{2} \eta^{\prime 2}}+\frac{1}{k^{4} \eta^{2} \eta^{\prime 2}}\right)\right. \\
& \left.+2 \delta_{\eta} \sin 2 k \delta_{\eta}\left(\frac{1}{k \eta \eta^{\prime}}+\frac{1}{k^{3} \eta^{2} \eta^{\prime 2}}\right)\right]
\end{aligned}
$$

where

$$
\delta_{\eta}=\eta-\eta^{\prime} .
$$

The noise kernel is clearly ultraviolet finite and therefore requires no renormalization. This should not be surprising. From (A30) we know that the kernels are built from the quadratic field operators $\psi^{2}$ rather than the linear operators $\psi$ of conventional two-point functions (which are well known to be ultraviolet divergent). The integrals in (A37) can be done by using the integral identities

$$
\begin{gathered}
\int \frac{e^{i k x}}{k^{m}} d k=\frac{1}{m-1}\left[-\frac{e^{i k x}}{k^{m-1}}+i x \int \frac{e^{i k x}}{k^{m-1}} d k\right] \\
\int \frac{e^{i k x}}{k} d k=\operatorname{Ci}(k x)+i \operatorname{Si}(k x)
\end{gathered}
$$

where $\mathrm{Si}$ and $\mathrm{Ci}$ are the sine and cosine integral functions . We then find that (A37) becomes 


$$
\begin{aligned}
\nu\left(s, s^{\prime}\right) & =\frac{H^{4}}{128 \pi^{6}} e^{6 H \sigma}\left[3 \pi \epsilon^{3} H^{-1} \delta_{d}(\delta)+\cos (4 \epsilon \sinh (H \delta / 2))\left(2+12 \epsilon^{2}+8 \epsilon^{2} \sinh ^{2}(H \delta / 2)\right)\right. \\
& +\sin (4 \epsilon \sinh (H \delta / 2))\left(8 \epsilon \sinh (H \delta / 2)-\frac{3 \epsilon^{3}}{2} \sinh ^{-1}(H \delta / 2)\right) \\
& \left.+16 \epsilon^{3} \operatorname{Si}(4 \epsilon \sinh (H \delta / 2))\left(3 \sinh (H \delta / 2)+2 \sinh ^{3}(H \delta / 2)\right)\right]
\end{aligned}
$$

where $\delta_{d}$ denotes the Dirac delta function which is not to be confused with $\delta$ defined in (3.10). This noise kernel does not diverge for $\epsilon \ll 1$ because the scaling (A34) makes the kernel proportional to $\Omega^{-1}$. Using (2.11) with $\Lambda=H a$ we find that

$$
\Omega^{-1}=\frac{3 \epsilon^{3} H^{3} e^{3 H s}}{4 \pi^{4}}
$$

which shows that $\Omega^{-1}$ scales the kernel by $\epsilon^{3}$. The noise kernel (A41) has a correlation time which we will define as the time when the argument of the cosine in (A41) equals $\pi / 4$. We then find that the correlation timescale $\tau_{c}$ is

$$
\tau_{c}=2 H^{-1} \ln (\pi / 8 \epsilon)
$$

The noise kernel is highly oscillatory for $\delta>\tau_{c}$. It is therefore effectively cutoff beyond the correlation time. We can therefore consider the approximate noise kernel

$$
\nu\left(s, s^{\prime}\right) \simeq \frac{H^{4}}{64 \pi^{6}} e^{6 H \sigma} \cos (4 \epsilon \sinh (H \delta / 2))+O\left(\epsilon^{2}\right), \quad \epsilon \ll 1 \text { and } \delta<\tau_{c} .
$$

Clearly the noise kernel has simplified greatly. However what is most pleasing is that the noise kernel has lost its algebraic dependence on $\epsilon$. Because the noise kernel is ultraviolet finite we didn't impose the inflationary cutoff. Had we done so the cutoff would have contributed terms of order $\epsilon^{3}$ into the noise kernel. Thus in the small $\epsilon$ limit the noise kernel is also independent of any ultraviolet cutoff.

We will now consider the dissipation kernel (A29). The scaling (A34) eliminates the volume factor $\Omega$ from the kernel in (A29) which upon substituting (A35) becomes

$$
\begin{aligned}
\mu\left(s, s^{\prime}\right) & =\frac{a(s) a\left(s^{\prime}\right)}{8 \pi^{2}} \int_{\epsilon H a}^{H a} d k\left[\sin 2 k \delta_{\eta}\left(1+\frac{2}{k^{2} \eta \eta^{\prime}}-\frac{\delta_{\eta}^{2}}{k^{2} \eta^{2} \eta^{\prime 2}}+\frac{1}{k^{4} \eta^{2} \eta^{\prime 2}}\right)\right. \\
& \left.-2 \delta_{\eta} \cos 2 k \delta_{\eta}\left(\frac{1}{k \eta \eta^{\prime}}+\frac{1}{k^{3} \eta^{2} \eta^{\prime 2}}\right)\right] .
\end{aligned}
$$

All of the terms in the above integral are ultraviolet finite except for the first flat space contribution which leads to a logarithmic divergence. For this reason we have used the inflationary ultraviolet cutoff. Using the integrals (A39-40) we find that (A45) becomes

$$
\begin{aligned}
\mu\left(s, s^{\prime}\right) & =\frac{H}{48 \pi^{2}} e^{3 H \sigma}\left[\sin (4 \epsilon \sinh (H \delta / 2))\left(2 \epsilon^{-3}+12 \epsilon^{-1}+8 \epsilon^{-1} \sinh ^{2}(H \delta / 2)\right)\right. \\
& +\cos (4 \epsilon \sinh (H \delta / 2))\left(\frac{3}{2} \sinh ^{-1}(H \delta / 2)-8 \epsilon^{-2} \sinh (H \delta / 2)\right) \\
& -16 \operatorname{Ci}(4 \epsilon \sinh (H \delta / 2))\left(3 \sinh (H \delta / 2)+2 \sinh ^{3}(H \delta / 2)\right)
\end{aligned}
$$




$$
\begin{aligned}
& -\sin (4 \sinh (H \delta / 2))\left(14+8 \sinh ^{2}(H \delta / 2)\right) \\
& -\cos (4 \sinh (H \delta / 2))\left(\frac{3}{2} \sinh ^{-1}(H \delta / 2)-8 \sinh (H \delta / 2)\right) \\
& \left.+16 \mathrm{Ci}(4 \sinh (H \delta / 2))\left(3 \sinh (H \delta / 2)+2 \sinh ^{3}(H \delta / 2)\right)\right] .
\end{aligned}
$$

We are only interested in the dissipation kernel for $\delta<\tau_{c}$. For times greater than this the dissipation kernel is highly oscillatory and therefore effectively cutoff. In this regime we can use the approximations

$$
\cos x \simeq 1-x^{2} / 2, \quad \sin x \simeq x-x^{3} / 3
$$

to get the leading order approximation to the dissipation kernel (A46) which is

$$
\begin{aligned}
\mu\left(s, s^{\prime}\right) & \simeq \frac{H}{\pi^{2}} e^{3 H \sigma}\left[\frac{1}{32} \sinh ^{-1}(H \delta / 2)+\sinh (H \delta / 2)+\frac{10}{9} \sinh ^{3}(H \delta / 2)\right. \\
& -\operatorname{Ci}(4 \epsilon \sinh (H \delta / 2))\left(\sinh (H \delta / 2)+\frac{2}{3} \sinh ^{3}(H \delta / 2)\right) \\
& -\sin (4 \sinh (H \delta / 2))\left(\frac{7}{24}+\frac{1}{6} \sinh ^{2}(H \delta / 2)\right) \\
& -\cos (4 \sinh (H \delta / 2))\left(\frac{1}{32} \sinh ^{-1}(H \delta / 2)-\frac{1}{6} \sinh (H \delta / 2)\right) \\
& \left.+\operatorname{Ci}(4 \sinh (H \delta / 2))\left(\sinh (H \delta / 2)+\frac{2}{3} \sinh ^{3}(H \delta / 2)\right)+O\left(\epsilon^{2}\right)\right], \quad \epsilon \ll 1 \text { and } \delta<\tau_{c} .
\end{aligned}
$$

Remarkably we find that in this approximation the dissipation kernel has lost its algebraic dependence on $\epsilon$.

Unfortunately the dissipation kernel can not be approximated to a simple expression like the noise kernel. However the slow roll conditions of inflation ensure that we need only consider an order of magnitude estimate of the integral

$$
\int_{s-\tau_{c}}^{s} d s^{\prime} \mu\left(s, s^{\prime}\right) \sim \tau_{c} \mu\left(s, s-\tau_{c}\right)
$$

which we obtain by multiplying the correlation time $\tau_{c}$ defined in (A43) by the dissipation kernel evaluated at $\delta=\tau_{c}$. From (A48) we can write

$$
\mu\left(s, s-\tau_{c}\right) \simeq \frac{10 H}{9 \pi^{2}} e^{3 H s} e^{-3 H \tau_{c} / 2} \sinh ^{3}\left(H \tau_{c} / 2\right) \simeq \frac{H}{8 \pi^{2}} e^{3 H s}
$$

where we used

$$
\sinh \left(H \tau_{c} / 2\right) \simeq e^{H \tau_{c} / 2} / 2
$$

Using (A43) and (A50) we find that (A49) becomes

$$
\int_{s-\tau_{c}}^{s} d s^{\prime} \mu\left(s, s^{\prime}\right) \sim \frac{e^{3 H s}}{4 \pi^{2}} \ln (\pi / 8 \epsilon)
$$




\section{REFERENCES}

[1] T. Padmanabhan, Structure Formation in the Early Universe, Cambridge University Press (1993); P.J.E. Peebles, Principles of Physical Cosmology, Princeton University Press, N.J. (1993); A.R. Liddle and D.H. Lyth, Phys. Rep. 231, 1 (1993); A.D. Linde, Particle Physics and Inflationary Cosmology, Harwood Academic Publishers GmbH, Chur, Switzerland, (1990); E.W. Kolb and M.S. Turner, The Early Universe, Addison Wesley (1990).

[2] A. Guth and S. Y. Pi, Phys. Rev. Lett. 49, 1110 (1982); A. A. Starobinsky, Phys. Lett. 117B, 175 (1982); S. W. Hawking, Phys. Lett. 115B, 295 (1982); J. M. Bardeen, P. J. Steinhardt and M. S. Turner, Phys. Rev. D28, 629 (1983); R. Brandenberger, R. Kahn and W. Press, Phys. Rev. D 28, 1809 (1983); V. Mukhanov, H. Feldman and R. Brandenberger, Phys. Rep. 215, 203 (1992); R. Brandenberger, Nucl. Phys. B. 245, 328 (1984); A. H. Guth and S. Y. Pi, Phys. Rev. D32, 1899 (1985).

[3] See, e.g., K. Lindenberg and B. J. West, The Nonequilibrium Statistical Mechanics of Open and Closed Systems (VCH Press, New York, 1990).

[4] M. Morikawa, Prog. Theor. Phys 93, 685 (1995).

[5] A. A. Starobinsky, in Field Theory, Quantum Gravity and Strings, ed. H. J. de Vega and N. Sanchez (Springer, Berlin 1986).

[6] S. J. Rey, Nucl. Phys. B284, 706 (1987); J. M. Bardeen and G. J. Bublik, Class. Quan. Grav. 4, 473 (1987); M. Morikawa, Phys. Rev. D42, 1027 (1990); H. E. Kandrup, Phys. Rev. 39, 2245 (1989)

[7] I. Yi and E. T. Vishniac, Phys. Rev. D43, 5295 (1993); Phys. Rev. D45, 3441 (1992); D.S. Salopek and J.R. Bond, Phys. Rev. D43, 1005 (1991); I. Yi, E.T. Vishniac and S. Mineshige, Phys. Rev. D43, 362 (1991); S. Mollerach, S. Matarrese, A. Ortolan, and F. Lucchin, Phys. Rev. D44, 1670 (1991); G.V. Chibisov and Yu. V. Shtanov, Int. J. Mod. Phys. A13, 2625 (1990); D.S. Salopek, J.R. Bond and J.M. Bardeen, Phys. Rev. D40, 1753 (1989); S. Matarrese, A. Ortolan and F. Lucchin, Phys. Rev. D40, 290 (1989); A. Ortolan, F. Lucchin and S. Matarrese, Phys. Rev. D38, 462 (1988).

[8] A. Linde, D. Linde and A. Mezhlumian, Phys. Rev. D 49, 1783 (1994); Phys. Lett. B307, 25 (1993); Phys. Lett. B345, 203 (1995); Y. Nambu and M. Sasaki, Phys. Lett. B 219, 240 (1989); Y. Nambu, Prog. Theor. Phys 81, 1037 (1989).

[9] A. Hosoya, M. Morikawa and K. Nakayama, Int. J. Mod. Phys. A 4, 2613 (1989); Y. Nambu, Phys. Lett. B 276, 11 (1992); M. Mijic, Phys. Rev. D49, 6434 (1994).

[10] F.R. Graziani, Phys. Rev. D38, 1122 (1988);D38, 1131 (1988);D38, 1802 (1988); D39, 3630 (1989);O.E. Buryak, Phys. Rev. D 53, 1763 (1996).

[11] This is discussed by J. R. Ray, Am. J. Phys. 47, 626 (1979).

[12] B. L. Hu and Y. Zhang, "Coarse-Graining, Scaling, and Inflation" Univ. Maryland Preprint 90-186 (1990); B. L. Hu, in Relativity and Gravitation: Classical and Quantum Proceedings of SILARG VII, Cocoyoc, Mexico, Dec. 1990. eds. J. C. D' Olivo et al (World Scientific, Singapore, 1991).

[13] M. Morikawa, in Quantum Physics and the Universe, ed. Namiki, K. Maeda, et al (Pergamon Press, Tokyo, 1993). Vistas in Astronomy, 37, 87 (1993).

[14] S. Habib, Phys. Rev. D46, 2408 (1992)

[15] E. Calzetta and B.L. Hu, Phys. Rev. D 52, 6770 (1995) 
[16] B. L. Hu, J. P. Paz and Y. Zhang, in The Origin of Structure in the Universe, ed. by E. Gunzig and P. Nardone (Kluwer, Dordrecht 1993).

[17] B. L. Hu, in Quantum Physics and the Universe, ed. Namiki, K. Maeda, et al (Pergamon Press, Tokyo, 1993). Vistas in Astronomy 37, 391 (1993).

[18] B. L. Hu, in Proc. Second International Workshop on Thermal Fields and Their Applications, eds. H. Ezawa et al (North-Holland, Amsterdam, 1991).

[19] F. Lombardo and F.D. Mazzitelli, Phys. Rev. D 53, 2001 (1996).

[20] B. L. Hu, J. P. Paz and Y. Zhang, Phys. Rev. D45, 2843 (1992); Phys. Rev. D47, 1576 (1993).

[21] R. Feynman and F. Vernon, Ann. Phys. (NY) 24, 118 (1963); R. Feynman and A. Hibbs, Quantum Mechanics and Path Integrals, (McGraw - Hill, New York, 1965); A.

O. Caldeira and A. J. Leggett, Physica 121A, 587 (1983); H. Grabert, P. Schramm and G. L. Ingold, Phys. Rep. 168, 115 (1988).

[22] V. Hakim and V. Ambegoakar, Phys. Rev. A 32, 423 (1985).

[23] B. L. Hu, in Quantum Mechanics in Curved Spacetime, ed. J. Audretsch and V. de Sabbata (Plenum, London 1990).

[24] S. Sinha, Ph. D. Thesis, University of Maryland (1991)

[25] J. P. Paz and S. Sinha, Phys. Rev. D44, 1038 (1991); ibid D45, 2823 (1992).

[26] B. L. Hu, J. P. Paz and S. Sinha,in Directions in General Relativity Vol. 1: Misner Festschrift eds B. L. Hu, M. P. Ryan and C. V. Vishveswara (Cambridge University Press, Cambridge, 1993).

[27] For earlier work on decoherence in quantum cosmology, see, e.g., C. Kiefer, Clas. Q. Grav. 4, 1369 (1987); J. J. Halliwell, Phys. Rev. D39, 2912 (1989); T. Padmanabhan, ibid. 2924 (1989).

[28] B. L. Hu, Physica A158, 399 (1989).

[29] B. L. Hu, in Proceedings of the Third International Workshop on Thermal Field Theory and Applications, ed. R. Kobes and G. Kunstatter (World Scientific, Singapore, 1994).

[30] B. L. Hu and Y. Zhang, in Proceedings of the Third International Workshop on Quantum Nonintegrability, eds J. M. Yuan, D. H. Feng and G. M. Zaslavsky (Gordon and Breach, Langhorne, 1993); Mod. Phys. Lett. A8, 3575 (1993).

[31] J.J. Halliwell, Phys. Rev. D48, 2739 (1993); A. Anderson and J. J. Halliwell, Phys. Rev. D48, 2753 (1993); J.J. Halliwell, Phys. Rev. D48, 4785 (1993).

[32] W. H. Zurek, Phys. Rev. D24, 1516 (1981); D26, 1862 (1982); in Frontiers of Nonequilibrium Statistical Physics, ed. G. T. Moore and M. O. Scully (Plenum, N. Y., 1986); Physics Today 44, 36 (1991); E. Joos and H. D. Zeh, Z. Phys. B59, 223 (1985); A. O. Caldeira and A. J. Leggett, Phys. Rev. A31, 1059 (1985); W. G. Unruh and W. H. Zurek, Phys. Rev. D40, 1071 (1989); B. L. Hu, J. P. Paz and Y. Zhang, Phys. Rev. D45, 2843 (1992); D47, 1576 (1993); W. H. Zurek, J. P. Paz and S. Habib, Phys. Rev. Lett. 47, 1187 (1993); J. P. Paz, S. Habib and W. H. Zurek, Phys. Rev. D47, 488 (1993); J. P. Paz and W. H. Zurek, Phys. Rev. D48, 2728 (1993).

[33] R. B. Griffiths, J. Stat. Phys. 36, 219 (1984); R. Omnés, J. Stat Phys. 53, 893, 933, 957 (1988); Ann. Phys. (NY) 201, 354 (1990); Rev. Mod. Phys. 64, 339 (1992).

[34] M. Gell-Mann and J. B. Hartle, in Complexity, Entropy and the Physics of Information, ed. by W. H. Zurek (Addison-Wesley, Reading, 1990); H. F. Dowker and J. J. Halliwell, Phys. Rev. D46, 1580 (1992); A. Albrecht, Phys. Rev. D48, 3768 (1993); J. Twamley, 
Phys. Rev. D48 (1993).

[35] M. Gell-Mann and J. Hartle, Phys. Rev. D47, 3345 (1993); T. Brun, Phys. Rev. D47, 3383 (1993).

[36] E. Calzetta and B. L. Hu, in Directions in General Relativity, Vol II: Brill Festschrift eds B. L. Hu and T. A. Jacobson (Cambridge University Press, Cambridge, 1993).

[37] W. H. Zurek, Prog. Theor. Phys. 89, 281 (1993).

[38] J. B. Hartle, Les Houches 1992 Lectures (1993). J. B. Hartle, in Directions in General Relativity, Vol. 1: Misner Festschrift eds B. L. Hu, M. P. Ryan and C. V. Vishveswara (Cambridge Univ., Cambridge, 1993).

[39] R. Omnés, Rev. Mod. Phys. 64, 339 (1992).

[40] B. L. Hu and A. Matacz, Phys. Rev. D49, 6612 (1994).

[41] B. L. Hu and A. Matacz, Phys. Rev. D 51, 1577 (1995).

[42] L. Grishchuk and Y. V. Sidorov, Phys. Rev. D42, 3414 (1990); A. Matacz, Phys. Rev. D49, 788 (1994).

[43] A.J. McKane, H.C. Luckock and A.J. Bray, Phys. Rev. A 41, 644 (1990); A.J. McKane, A.J. Bray and T. Newman, Phys. Rev. A 41, 657 (1990); H.C. Luckock and A.J. McKane, Phys. Rev. A 42, 1982 (1990). 